\title{
Nasopharyngeal Masses Arising from Embryologic Remnants of the Clivus: A Case Series
}

\author{
Mirabelle Sajisevi $^{1}$ Jenny K. Hoang ${ }^{2}$ Rose Eapen ${ }^{1}$ David W. Jang ${ }^{1}$ \\ ${ }^{1}$ Division of Head and Neck Surgery \& Communication Sciences, \\ Department of Surgery, Duke University Medical Center, Durham, \\ North Carolina, United States \\ 2 Deparment of Radiology, Duke University Medical Center, Durham, \\ Address for correspondence Mirabelle Sajisevi, MD, Division of \\ Otolaryngology-Head and Neck Surgery, Department of Surgery, \\ Duke University Medical Center, DUMC 3805 Durham, NC, 27710, \\ United States (e-mail: Mirabelle.sajisevi@dm.duke.edu).
} North Carolina, United States

J Neurol Surg Rep 2015;76:e253-e257.

\section{Introduction}

The differential diagnosis of nasopharyngeal masses is broad, with the most common being nasopharyngeal carcinoma. However, one special category of nasopharyngeal masses to consider is tumors originating from the clivus. The clivus, which includes a portion of the occipital and sphenoid bone, comprises the posterior border of the nasopharynx, and is an area that contains a complex array of embryologic remnants. Each of these remnants can give rise to a nasopharyngeal mass with only subtle involvement of the clival bone. Careful examination of imaging studies may provide diagnostic clues for the clinician. Moreover, the embryologic origin of each tumor has important implications for treatment. In this case series, we discuss the clinical and imaging characteristics of patients with infrasellar craniopharyngioma, intranasal received

February 14, 2015 accepted after revision

August 8, 2015

published online

October 25, 2015
Dol http://dx.doi.org/

10.1055/s-0035-1564603. ISSN 2193-6366. (c) 2015 Georg Thieme Verlag KG
Stuttgart . New York

License terms

(요 (1) $\Theta \circledast$ 
extraosseous chordoma, and canalis basilaris medianus. We also review the embryology of the clivus to explain the pathogenesis of these entities.

\section{Methods}

Approval was obtained from the Duke University Medical Center Institutional Review Board. This is a case series of three patients. Each patient was evaluated and treated at the Duke University by the two senior authors (R. E. and D.W. J.). Clinical presentation, computed tomography (CT), and magnetic resonance imaging (MRI) characteristics, and treatment are discussed. A brief review of each lesion, as well as the embryology of the clivus is also presented (-Fig. 1).

\section{Results}

\section{Case 1: Infrasellar Craniopharyngioma}

A 16-year-old male patient with a history of craniopharyngioma resection at an outside institution several years earlier presented with nasal obstruction. On nasal endoscopy, there was a mass filling the nasopharynx and right posterior nasal cavity. CT and MRI revealed a large peripherally enhancing cystic mass occupying the nasopharynx and the right pterygopalatine fossa (-Fig. 2). There was no bony destruction of the clivus, but on CT the mass abutted a patent craniopharyngeal canal (-Fig. 2). Gross total resection of the mass, as well as the craniopharyngeal canal, was performed through a combined transnasal and transantral endoscopic approach. Histologic examination revealed adamantinomatous craniopharyngioma.

\section{Case 2: Extraosseous Chordoma}

A 43-year-old male patient presented with a 1-year history of nasal obstruction and headache. On nasal endoscopy, there was a large polypoid mass filling the nasopharynx and the bilateral posterior nasal cavity. CT and MRI revealed a heterogeneously enhancing polypoid mass in the nasopharynx abutting the clivus without bony erosion or destruction (-Fig. 3). Intraoperatively, the mass was found to be origi-

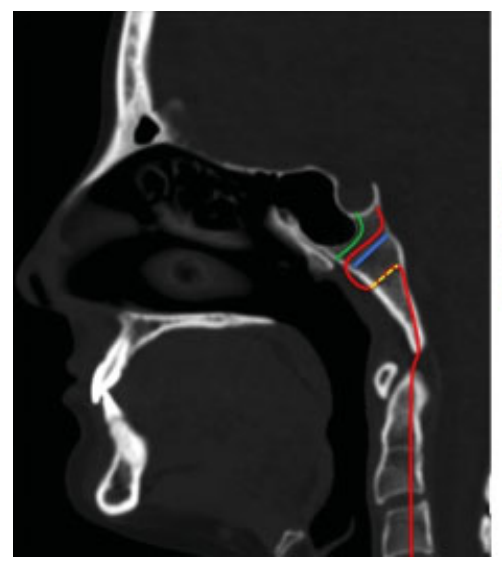

Craniopharyngeal canal

Notochord

Epheno-occiptal synchondrosis 12. Canalis basilaris medianus

Fig. 1 Diagram of embryological structures of the clivus. (Image adapted from Galán et al. ${ }^{11}$ )
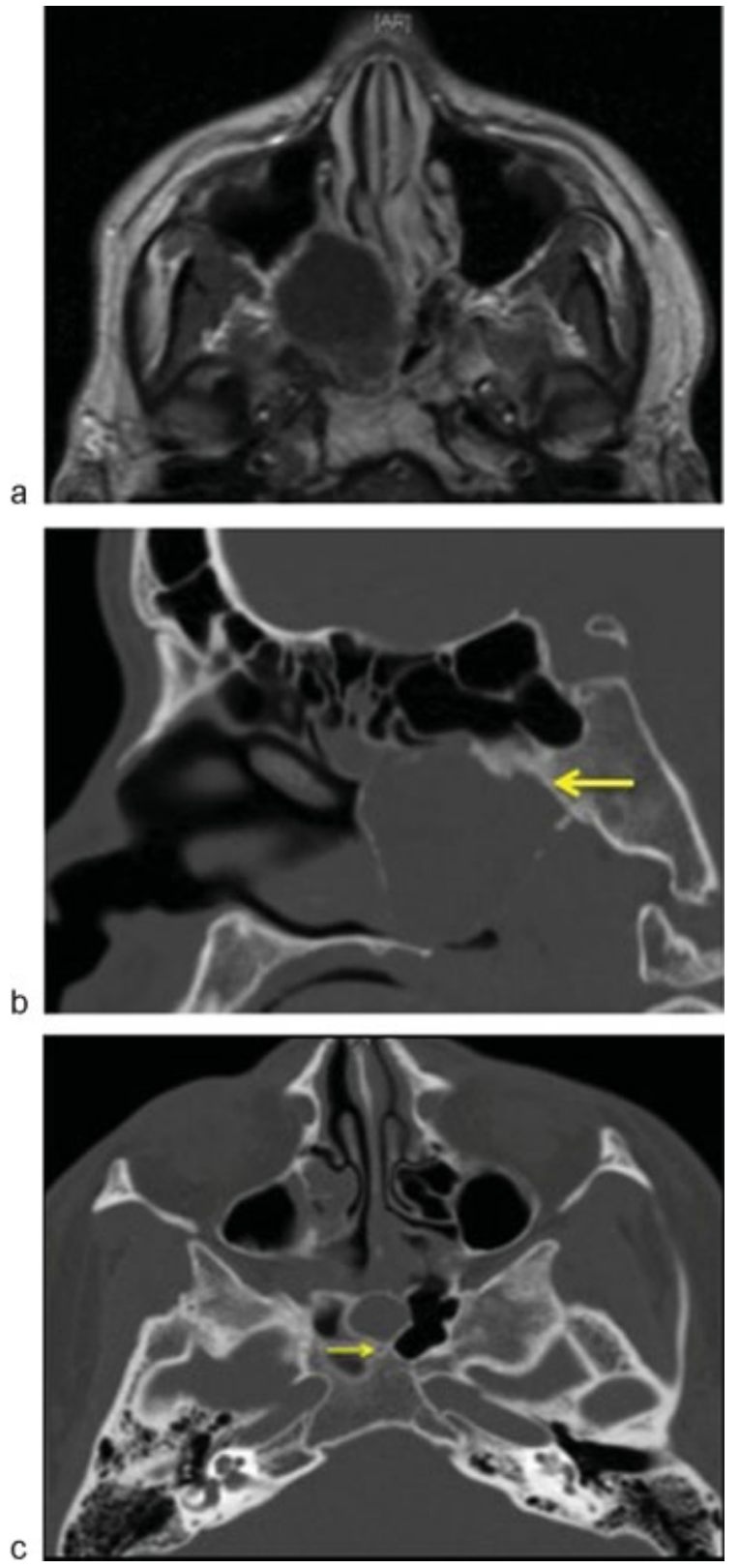

Fig. 2 (Case 1) Imaging of an infrasellar craniopharyngioma: (a) An enhanced T1-weighted MRI shows a mass in the nasopharynx eccentric to the right with peripheral enhancement. (b) A sagittal CT image revealing minimal sclerosis and remodeling of the clivus (arrow) without bony destruction. (c) On an axial CT image shows a patent craniopharyngeal canal (arrow). CT, computed tomography; MRI, magnetic resonance imaging.

nating from the clivus. Gross total resection, which included drilling of the clival attachment site, was performed endoscopically. Histopathological analysis revealed chondroid chordoma. The patient was subsequently referred for adjuvant treatment with proton beam radiation.

\section{Case 3: Canalis Basilaris Medianus}

A 4-year-old female patient presented with a recurrent nasopharyngeal polyp. She initially underwent adenoidectomy and pressure equalization tube placement for 
a

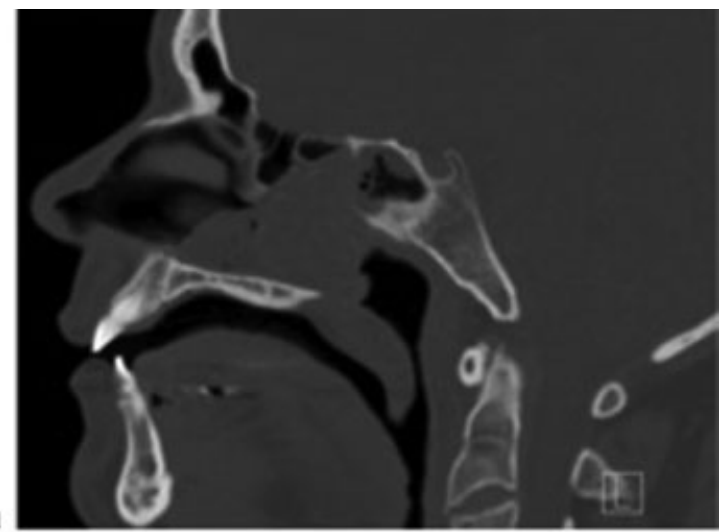

b
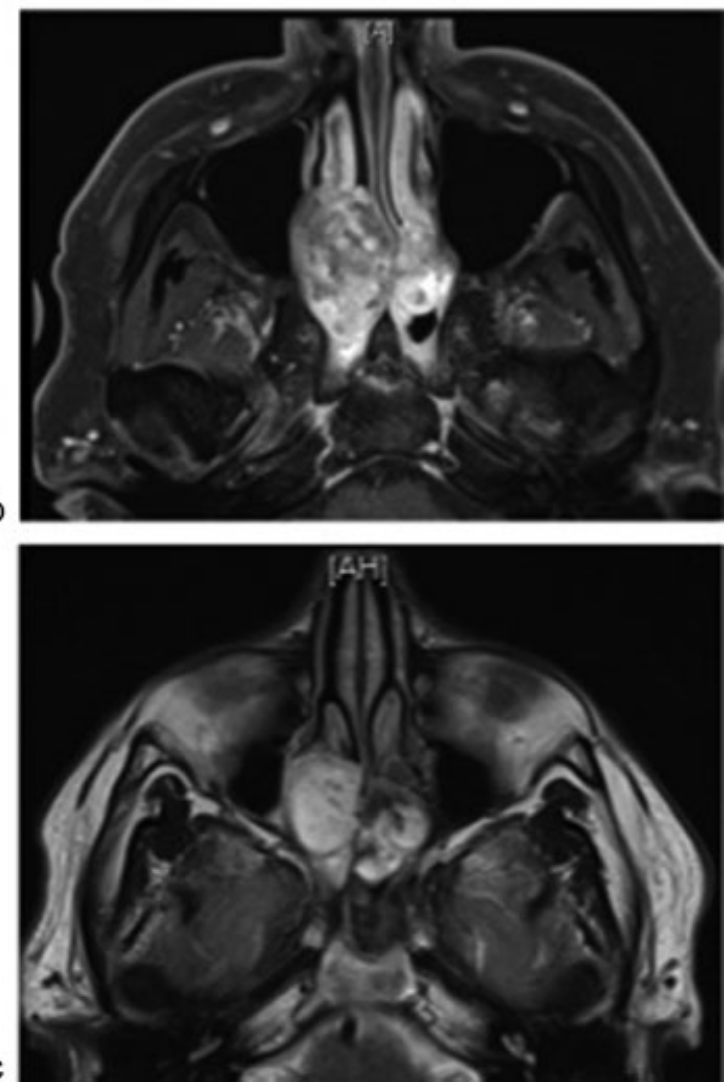

Fig. 3 (Case 2) Images of the intranasal extraosseous chordoma: (a) A sagittal $C T$ image showing mass abutting sphenoid sinus and clivus without bony erosion. (b) An enhanced axial T1-weighted MRI demonstrating a heterogeneously enhancing mass in the posterior nasal cavity and nasopharynx. (c) An axial T2-weighted MRI demonstrating a hyperintense mass in the nasal cavity and low-intensity fibrous septa. $\mathrm{CT}$, computed tomography; MRI, magnetic resonance imaging.

recurrent otitis media at an outside institution. After 2 months, she developed increased snoring and nasal congestion, and a nasopharyngeal polyp was noted and removed. Recurrence with resection of this polyp occurred three more times before she was referred to the Duke University Medical Center. She did not report rhinorrhea or meningitis. On examination, the adenoid pad was surgically resected, but there was a small, pale, polypoid nasopharyngeal mass. Because a meningocele was suspected, a CT cisternogram was performed, which revealed a soft tissue mass possibly emanating from a bony defect of the middle clivus suggestive of canalis basilaris medianus (-Fig. 4). However, there was no evidence of cerebrospinal fluid communication through this space on cisternogram or MRI. Following her final polypectomy, she did not experience any further clinical symptoms. There was no clinical or radiographical evidence for cerebrospinal fluid leak and no return of her nasopharyngeal polyposis.

\section{Discussion}

It is valuable to review the basic anatomy and embryology of the clivus. The clivus, which includes a portion of the occipital and sphenoid bone, slopes posteriorly from the base of the dorsum sellae to the foramen magnum. ${ }^{1}$ It develops through the endochondral bone formation, in which a cartilaginous framework first develops and then resorbs with subsequent deposition of bone. ${ }^{1,2}$ Elongation of the clivus occurs at the sphenooccipital synchondrosis which does not ossify until the age of 14 to 16 years. ${ }^{2}$ The craniopharyngeal canal, notochord, and the canalis basilaris medianus are transient structures under normal
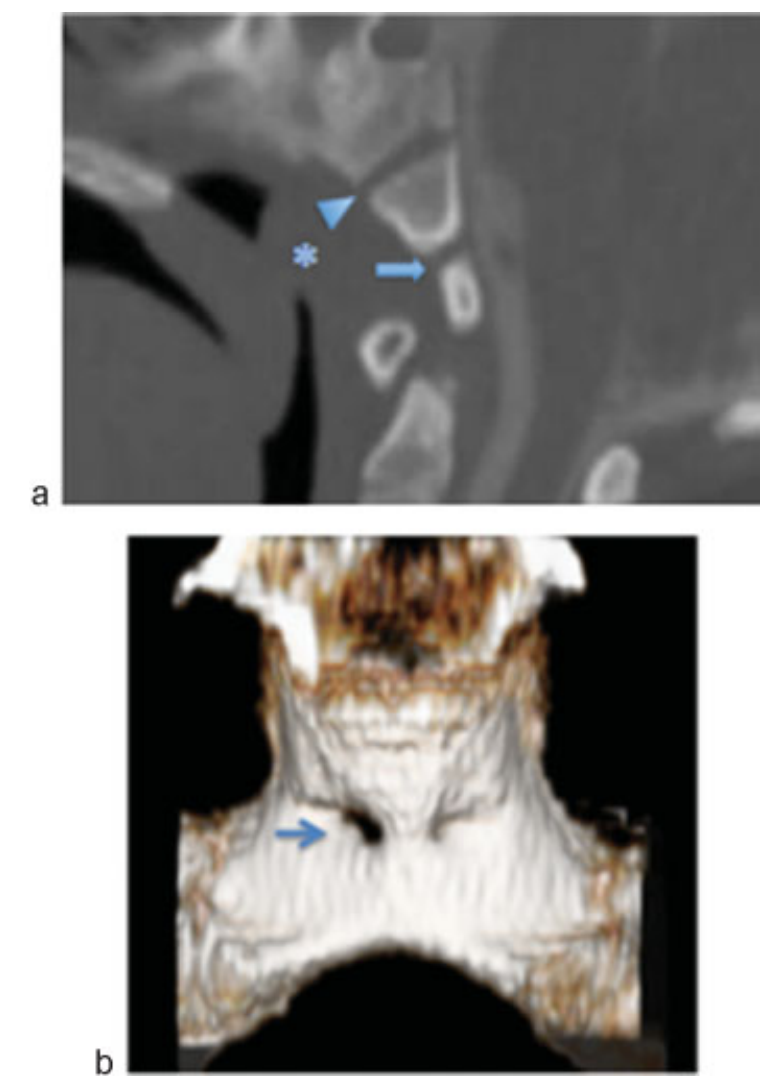

Fig. 4 (Case 3) Images of polypoid mass arising from the canalis basilaris medianus: (a) Sagittal CT scan showing a mass in the nasopharynx (asterisk) that abuts the canalis basilaris medianus (arrow) and the sphenooccipital synchondrosis (arrowhead). (b) A CT surface rendered reformatted image shows a patent canalis basilaris medianus (arrow). CT, computed tomography; MRI, magnetic resonance imaging. 
circumstances, but our three cases show pathology associated with persistence of these structures.

The craniopharyngeal canal is related to the development of the pituitary gland. Rathke pouch, which forms the anterior lobe of the pituitary gland, arises from the primitive stomodeum and extends cranially through the clivus toward the neural ectoderm during the 4 th week of gestation, forming the craniopharyngeal canal. ${ }^{3}$ Normally, the craniopharyngeal canal is obliterated and the Rathke pouch loses its attachment with the stomodeum during the 8th week of gestation. ${ }^{3}$ The notochord is also closely associated with the clivus. It is a primitive cell line around which the skull base and axial skeleton develop. ${ }^{4}$ Rostrally, it courses intraclivally then exits ventrally into the nasopharyngeal soft tissues through the canalis basilaris medianus before returning to an intraclival location. The sphenooccipital synchondrosis is located in between the craniopharyngeal canal and the canalis basilaris medianus and its remnant is typically visible on CT imaging (-Fig. 1).

Each case in our series underscores the clinical significance of an embryologic remnant of the clivus. Craniopharyngiomas arise from cellular remnants of the Rathke pouch and have a bimodal distribution., ${ }^{5,6}$ In children and adolescents, the histologic type is usually adamantinomatous with cyst formation and calcifications. In adults, they are more likely to be solid with papillary architecture on histology. ${ }^{6}$ Because of their close association with the Rathke pouch, craniopharyngiomas are typically suprasellar. However, infrasellar craniopharyngiomas originate from an unobliterated craniopharyngeal canal, which extends from the floor of the sella to the vomer. ${ }^{3}$ They have been reported in the nasopharynx where they are purely extracranial, mimicking a sinonasal mass as in our case. ${ }^{3}$ They are less likely to demonstrate contrast enhancement and calcification, as compared with the typical suprasellar craniopharyngioma. ${ }^{3,7}$ Careful examination of the CT scan can demonstrate communication between the nasopharyngeal mass and a patent craniopharyngeal canal. Management should include identification and resection of the entire craniopharyngeal canal.

The second case, an intranasal extraosseous chordoma, also demonstrates the complex embryologic anatomy of the clivus. Chordomas are rare malignant tumors of notochordal origin that generally occur at the sphenooccipital or sacral region of the axial skeleton. ${ }^{4}$ Histologically, chordomas are categorized as classical, chondroid, or dedifferentiated. ${ }^{8}$ Chondroid chordomas are the least aggressive of the three, while the dedifferentiated type is faster growing and more likely to metastasize. ${ }^{8}$ Chordomas are typically intracranial and present as a soft tissue mass with bony destruction of the clivus. $^{9}$ On rare occasions, however, chordomas may arise in extraosseous locations such as the nasopharynx. ${ }^{4}$ This occurs because a portion of the notochord exits the medial basal canal and runs through the nasopharyngeal soft tissue. It is these extraosseous notochordal rests that are thought to give rise to intranasal extraosseous chordomas. ${ }^{4}$

On CT, chordomas appear as well-circumscribed, expansile soft tissue masses with irregular intratumoral calcifications.
On MRI, there is typically mild-to-moderate heterogeneous enhancement with "honeycomb" appearance. They also demonstrate hypointensity or isointensity on T1-weighted windows, and heterogeneous signal intensity on T2-weighted windows due to high fluid content of vacuolated cellular components. ${ }^{9}$ Fibrous septa can be seen as low signal intensity on T2 and are characteristic. ${ }^{9}$ As our case demonstrates, intranasal extraosseous chordomas may not exhibit any obvious involvement of the clivus on imaging. However, surgical resection should include the portion of the bony clivus at which this tumor originates.

The final case was clinically suspicious for a polyp arising from canalis basilaris medianus. The persistence of this structure is present in 2 to $3 \%$ of the patients. ${ }^{10}$ It is a welldefined channel originating on the intracranial surface of the midline basiocciput, thought to represent a passageway for remnants of the notochord or persistent emissary veins. These anatomical variants can be diagnosed on CT or MRI (-Fig.4). Although they can be associated with intracranial communication and subsequent meningitis, they should not be considered in themselves a pathological anomaly. ${ }^{10}$ If there is a nasopharyngeal mass in a patient with a persistent canalis basilaris medianus, one should consider the possibility of a meningocele. Usually these are small and managed conservatively. If surgery for a canalis basilaris medianus meningocele is necessary, one should prepare for potential repair of a cerebrospinal fluid leak.

\section{Conclusion}

Nasopharyngeal masses can arise from embryological remnants of the clivus. Understanding the embryological anatomy of the clivus and key imaging findings is essential for suspecting the differentials of infrasellar craniopharyngiomas, nasopharyngeal chordomas, and meningoceles of the canalis basilaris medianus.

Conflict of Interest

None.

Financial Disclosures

None.

\section{References}

1 Köksel T, Crockard A. "Clivus" Through the eyes of the transoral surgeon. Turk Neurosurg 1990;1:146-150

2 Hofmann E, Prescher A. The clivus: anatomy, normal variants and imaging pathology. Clin Neuroradiol 2012;22(2): 123-139

3 Nourbakhsh A, Brown B, Vannemreddy P, Lian T, Nanda A, Guthikonda B. Extracranial infrasellar ectopic craniopharyngioma: a case report and review of the literature. Skull Base 2010; 20(6):475-480

4 Nguyen RP, Salzman KL, Stambuk HE, Ahuja AT, Harnsberger HR. Extraosseous chordoma of the nasopharynx. AJNR Am J Neuroradiol 2009;30(4):803-807 
5 Russel DS, Rubinstein LJ. Pathology of Tumors of the Nervous System. 7th ed. Boca Raton, FL: Taylor and Francis; 2006:723

6 Müller HL. Childhood craniopharyngioma-current concepts in diagnosis, therapy and follow-up. Nat Rev Endocrinol 2010;6(11): 609-618

7 Fitz CR, Wortzman G, Harwood-Nash DC, Holgate RC, Barry JF, Boldt DW. Computed tomography in craniopharyngiomas. Radiology 1978;127(3):687-691

8 Nibu Y, José-Edwards DS, Di Gregorio A. From notochord formation to hereditary chordoma: the many roles of Brachyury. Biomed Res Int 2013;2013(13):826435
9 Yan ZY, Yang BT, Wang ZC, Xian JF, Li M. Primary chordoma in the nasal cavity and nasopharynx: CT and MR imaging findings. AJNR Am J Neuroradiol 2010;31(2):246-250

10 Jacquemin C, Bosley TM, al Saleh M, Mullaney P. Canalis basilaris medianus: MRI. Neuroradiology 2000;42(2): 121-123

11 Galán SL, Díaz MM, Soler RM, et al. Notochord pathology in the cranio-cervical junction: chordomas. Ecchordosis physaliphora vs chordomas with low radiological agressivity: a diagnostic challenge. Poster presented at: Annual Meeting of the European Congress of Radiology; March 6-10, 2014; Vienna, Austria. Poster C-2116 